Der Verfasser entwirft auf der Basis des vor kurzem erschienenen Rechenschaftsberichtes «La Géographie Française au milieu du XX $X^{\mathbf{e}}$ siècle» (Paris 1957) einen Rück- und Ausblick auf das moderne geographische Schaffen in Frankreich. Die Bilanz zeigt, daß die französischen Geographen auf so gut wie allen Gebieten ihres Faches Bemerkenswertes geleistet haben, in der allgemeinen Geographie wie in der Länderkunde, in den analytischen Bereichen wie in der Synthese, über heimatliche Themen wie über das europäische und außereuropäische Ausland. Obwohl bewußt die «Einheit»der Disziplin im Zentrum stand, war keineswegs Uniformität der einzelnen Arbeiten, sondern optimale Vielfalt die Folge. Dabei ist zweifellos - wie übrigens in andern Ländern — die Tendenz zu verstärkter Pflege der Anthropogeographie im weitesten Sinne (Wirtschaftsgeographie, Politische Geographie, Religionsgeographie, Sozialgeographie, Industriegeographie, Stadtgeographie usw.) zu erkennen, die sich auch im regionalen Sektor deutlich ausprägt. $\mathrm{Da} B$ in der Tat von einer Progression gesprochen werden kann, verdeutlichen in der Berichtszeit neu entstandene Institutionen wie das Nationalzentrum für wissenschaftliche Forschung, das Zentrum für kartographische und geographische Dokumentation sowie diverse Zeitschriften, die auch die starke Spezialisation erkennen lassen, die ähnlich wie andere Disziplinen neuerdings die Geographie durchdringt.

\title{
ZUR SYSTEMATIK DES TROPENKLIMAS
}

\section{Paul Schaufelberger}

\section{EINLEITUNG}

Es mag weite Kreise überraschen, daß man um die Mitte des 20. Jahrhunderts hinsichtlich der Beurteilung des Tropenklimas noch zu keiner befriedigenden einheitlichen Lösung gelangt ist. Doch stößt man trotz der bekannten Klimatatsachen auf folgende teils sich widersprechende Ansichten.

A. Hinsichtlich der Klimafaktoren und -elemente zeigt sich:

1. Grenzen der Tropen.

a) Mathematisch werden die Tropen durch die Wendekrcise begrenzt.

b) Zweifellos liegen in der Nähe dieser Parallelkreise bestimmte Jahresisothermen, wie diejenigen von 18 oder $20^{\circ}$, die ebenfalls die Grenze der Tropen erkennen lassen.

2. Temperaturen.

a) Infolge der Begrenzung der Tropen durch bestimmte Jahresisothermen hat man sich verführen lassen, die Tropen als heisse Klimazone zu definieren.

b) Bekannt ist aber auch die Tatsache der Gleichförmigkeit der mittleren Monatstemperaturcn, ganz unabhängig von deren absoluten Höhc. Dies trifft für die heisscn und kühlen Tropen zu.

3. Niederschlag.

a) Nach der Regenverteilung werden trockcuc, wechsclfcuchtc und immerfeuchte Tropen unterschieden.

b) Nach der Regenmenge werden die Tropen als arid, scmiarid, semihumid, humid und perhumid beurteilt.

Jeder, der sich mit den Tropen wissenschaftlich befaßt, hat sich daher zu entscheiden, welchen grundsätzlichen Standpunkt or wählen will. Je nach persönlicher Erfahrung oder dem Glauben an gewisse Autoritäten wird er die obigen Klimaelemente so oder so gruppieren. Die Folge sind 2.2. 2. $=8$ verschiedene Hypothesen hinsichtlich der Tropenklimate.

Naturgemäß dürfte nur cine Auffassung die richtige sein, so daß mindestens 7 Arbeitshypothesen sich als revisionsbedürftig erweisen. Kommt nun ein Beobachter, der sich einer dieser revisionsbedürftigen Annahme verschrieben hat, aus der gemäßigten Zone in die Tropen, so wird er andere Verhältnisse antreffen, als er erwartete. Er ist dann enttäuscht und kann sich nicht zurechtfinden. Er kommt leicht zum Schluß, daß die Klimavcrhältnisse in den Tropen anders geartet sind, als wie er sie sich im Vergleich mit der gemäßigten Zone vorstcllte. Er ist deshalb überzeugt, daß zur Erforschung der Tropen spezielle Arbeitsmethoden notwendig sind. Daraus ergibt sich ein weiteres Widerspruchspaar hinsichtlich

B. Hinsichtlich der Arbeitsmethode zeigt sich:

1. In den Tropen gelten dieselben Methoden wie in den übrigen Klimabereichen, d.h. man mu $\beta$ parallelisieren.

2. Die Erforschung der Tropen erfordert spezielle Arbeitsmethoden, d. h. man darf nicht vergleichen oder parallelisieren. 
Damit erhöht sich dic Zahl der «Tropenklimatologicn» von $\delta$ auf 16 ! M. a. W., die Tropenwerden klimatisch mindestens nach 16 Gesichtspunkten betrachtet, beschrieben, gedeutet, gelesen und systematisiert, während doch nur eine alle Elemente berücksichtigende Betrachtung den Tatsachen gerecht wird. Es ist begreiflich, daß viele Originalberichte von manchen Lesern nicht verstanden werden, weil sie in anderen «Hypothesen» denken!

Wollen wir aus dieser Situation herauskommen, so müssen wir das Übel an der Wurzel packen und bei jedem Widerspruchspaar untersuchen, welche Ansicht jeweils den heute bekannten Tatsachen gerecht wird!

\section{DIE TEMPERATUREN}

Die Monatsmittcl. Betrachten wir die mittleren Monatstemperaturen einiger Orte von Ecuador, in Mereshöhen von o bis etwa $3000 \mathrm{~m}$ !

Tab. 1. - Mittlere Monatstemperaturcn in Ecuador, in ${ }^{\circ} \mathrm{C}$

\begin{tabular}{lrrrrrrr} 
Monat & \multicolumn{3}{c}{ Guayaquil Arahumo La Toma Loja Shell Mora } & Baños & Ambato & Tulcán \\
\hline Januar & 25,9 & 24,4 & 23,3 & 22,5 & 16,8 & 14,2 & 10,7 \\
Februar & 25,9 & 24,9 & 23,1 & 22,4 & 16,6 & 14,8 & 11,2 \\
März & 26,7 & 24,7 & 24,5 & 21,8 & 16,8 & 14,1 & 10,8 \\
April & 26,8 & 24,4 & 23,5 & 22,2 & 16,7 & 14,5 & 11,1 \\
Mai & 26,1 & 25,0 & 23,6 & 21,4 & 16,3 & 14,3 & 11,4 \\
Juni & 25,8 & 24,4 & 24,4 & 21,5 & 15,9 & 13,7 & 11,1 \\
Juli & 24,4 & 23,9 & 23,3 & 20,5 & 15,3 & 12,5 & 10,4 \\
August & 25,0 & 25,0 & 24,0 & 20,7 & 15,0 & 12,6 & 10,0 \\
September & 25,3 & 24,4 & 24,5 & 21,3 & 16,2 & 13,6 & 9,9 \\
Oktober & 26,0 & 25,2 & 23,9 & 21,8 & 16,8 & 14,9 & 11,7 \\
November & 26,3 & 25,4 & 23,1 & 22,2 & 17,7 & 14,8 & 11,3 \\
Dezember & 26,9 & 25,1 & 22,9 & 21,9 & 17,2 & 14,2 & 11,4 \\
\hline Jahr & 25,9 & 24,7 & 23,7 & 21,7 & 16,4 & 14,0 & 10,9 \\
Maximum-Minimum & 2,5 & 1,5 & 1,6 & 2,0 & 2,7 & 2,4 & 1,8
\end{tabular}

Der Temperaturverlauf. Tab. 1 bestätigt die längst bekannte Tatsache der Gleichförmigkeit der mittleren Monatstemperaturen in den Tropen und ihre Unabhängigkeit von der absoluten Höhe der Jahrestemperaturen. In Ecuador liegen die Differenzen der extremsten Monatsmittel unter $3^{\circ} \mathrm{C}$. Weiter ist bekannt, daß die täglichen Temperaturschwankungen innerhalb der Wendekreise im freien Feld 12 bis $14^{\circ} \mathrm{C}$ betragen können. Der Temperaturablauf der Tropen ist somit durch geringe jahreszeitliche und starke tägliche Schwankungen charakterisiert. Kennzeichen des Tropenklimas sind daher: gleichförmige mittlere Monatstemperaturen, annähernd ständige Tag- und Nachtgleiche und deshalb als Jahreszeiten Regen- und Trockenzeiten, während die gemäßigten Zonen bekanntlich größere jahreszeitliche Temperaturunterschiede mit warmen Sommern und kalten Wintern haben. Noch ausgeprägtere Oszillationen beherrschen die Polargebiete, wo die Tageslänge zwischen 0 bis 24 Stunden liegt, so daß hier die Jahreszeiten Polarnacht und Polartag sind.

Wo liegen nun die Grenzen, wo die astronomisch bedingten Jahreszeiten die Namen wechseln? Die Schwankungen der monatlichen Temperaturmittel und Tageslängen sind Funktionen der geographischen Breite, so daß die Wende-und Polarkreise die Tropen, gemäßigte Zone und Polargebiete mathematisch in die alten geographischen Zonen gliedern, wohl auch mehr oder weniger die natürlichen Grenzen der Klimazonen sind. Da die Unterschiede zwischen den extremsten Monatsmitteln und der Tagslängen Funktionen der geographischen Breiten sind, so ist anzunehmen, daß die Differenzen längs der Wende- und Polarkreise in gewissen Grenzen liegen. Dort dürften auch gewisse Jahresisothermen $\mathrm{zu}$ finden sein, sofern die tatsächlichen Jahrestemperaturen auf Meereshöhe reduziert werden. Da die zonalen Klimate allmählich ineinander übergehen, so werden Wende- und Polarkreise, bestimmte Differenzen der extremsten mittleren Monatstemperaturen oder der Tageslänge sowie bestimmte Jahresisothermen als Grenzen verwendet werden können.

1 Differenz zwischen dem Mittel des wärmsten und kältesten Monats. 
Durch diese Grenzen sind aber die Tropenklimate nicht definiert. Das Tropenklima ist ein Zonalklima, das als Funktion der geographischen Breite der Verlauf der mittleren Monatstemperaturen und die Schwankungen der Tageslänge kennzeichnen.

Die mittleren Jahrestemperaturen. Aus Tab. 1 erkennen wir weiter, daß die mittleren Jahrestemperaturen von $25,9^{\circ}$ in Guayaquil auf $10,9^{\circ}$ in Tulcán sinken. Diese Temperaturabnahme erklärt sich aus den Höhendifferenzen, die sowohl in den Tropen wie in der gemäßigten Zone pro $100 \mathrm{~m}$ rund $0,6^{\circ} \mathrm{C}$ beträgt.

Wir können darum die Zonenklimate mittels der mittleren Jahrestemperaturen in Höhenklimate unterteilen. In Südamerika unterscheidet man, wie bekannt, folgende tropischen Höhenklimate:
1. Tierra caliente $\left(\mathrm{T}\right.$ über $\left.24^{\circ} \mathrm{C}\right)$;
4. Páramo ( $\left.\mathrm{T} 12-6^{\circ}\right)$ und
2. Tierra templada $\left(\mathrm{T} 2+-18^{\circ}\right)$;
5. Tierra helada ( $\mathrm{T}$ unter $6^{\circ}$ ).
3. Tierra fría ( $\left.\mathrm{T} 18-12^{\circ}\right)$;

Definiert man dagegen die Tropen durch die Jahresisothermen von $20^{\circ} \mathrm{C}$, so bereitet $z$. B. die Klimaklassifikation von Baño, Ambato und Tulcán unüberwindliche Schwierigkeiten. Tropisch sind sie nicht, weil die Jahrestemperaturen unter $20^{\circ} \mathrm{C}$ liegen. Der gemäßigten Zone gehören sie aber auch nicht an, weil ihre Jahreszeiten Regen- und Trockenzeiten und nicht warme Sommer und kalte Winter sind!

\section{NIEDERSCHLAG}

Regenverteilung. $\mathrm{Da}$ in den gemäßigten Zonen die Ansicht verbreitet ist, daß die Regenverteilung die trockenen, wechselfeuchten und immerfeuchten Tropen bestimme, prüfen wir erst diese Frage. Unter jahreszeitlicher Regenverteilung versteht man in der gemäßigten Zone den Anteil jedes Monats an der Jahresregenmenge. Auf die Tropen angewendet gehörten die Monate mit mehr als $81 / 3 \%$ der Jahresmenge zur Regenzeit. Wir können jedoch vereinfachend die Monate mit mehr als $1 / 12$ des Jahresniederschlages der Regenzeit zuteilen.

Danach ergibt sich leicht die Regenverteilung in Costa Rica, Mittelkolumbien und Flores (Tab.2). Da diese Gebiete verschiedener geographischer Breite angehören, muß sich zeigen, ob diese auf die Regenverteilung einen Einfluß ausübt.

Tab. 2. - Regenvertcilung in Costa Rica, Mittclkolumbicn und Flores, in cm

\begin{tabular}{|c|c|c|c|c|c|c|c|c|c|c|c|c|c|c|}
\hline Costa Rica & $\mathrm{J}$ & $\mathrm{F}$ & M & A & $\mathrm{M}$ & $\mathrm{J}$ & $\mathrm{J}$ & A & S & O & $\mathrm{N}$ & D) & & Trocl \\
\hline Caracol & 20 & 11 & 23 & 31 & 57 & 61 & 56 & 65 & 73 & 89 & 63 & 32 & & \\
\hline San José & 1 & o & 1 & 3 & 24 & 27 & 23 & 28 & 36 & 31 & 22 & 6 & 2 & \\
\hline Cartago & 5 & 3 & 1 & 1 & 20 & 17 & 11 & 13 & 17 & 26 & 15 & 8 & 1 & \\
\hline \multicolumn{15}{|c|}{ Mittelkolumbien } \\
\hline Dagua & 23 & 15 & 32 & 46 & 70 & 44 & 29 & 29 & tt & 68 & 61 & 15 & 4 & \\
\hline Manizales & 17 & 12 & 16 & 21 & 22 & 11 & 7 & 10 & 15 & 32 & 32 & 19 & & \\
\hline Cali & 9 & 8 & 11 & 14 & 16 & 9 & 2 & 4 & 9 & 13 & 12 & 15 & & \\
\hline \multicolumn{15}{|l|}{ Flores } \\
\hline Tœtend & 41 & 44 & 53 & 39 & 18 & 10 & 10 & 7 & 11 & 26 & 37 & 42 & & \\
\hline Badjawa & 42 & 38 & 31 & 19 & 10 & 4 & 6 & 3 & 2 & 5 & 17 & 37 & 2 & \\
\hline Reo & 28 & 35 & 20 & 6 & 4 & 2 & 2 & 2 & 1 & 2 & 5 & 19 & 1 & \\
\hline
\end{tabular}

Hieraus ergibt sich:

1. Die Regenverteilung ist abhängig von der geographischen Breite.

2. Die Tropische Regenzeit fällt mit dem höchsten Sonnenstand zusammen. Sie ist eine Jahreszeit, die in den äußeren Tropen (Costa Rica und Flores) in den astronomischen Sommer der betreffenden Hemisphäre fällt; in den innern Tropen treten zwei Regenzeiten auf: im Frühling und Herbst (DE MARTONN'sche Regel). 
3. Die sogen. tropische Trockenzeit ist nicht unbedingt eine regenarme (Trokken)- Periode (Caracol, Dagua, Manizales und Tatend).

4. Die Regenverteilung ist unabhängig vom Jahresniederschlag.

Wollen wir die Trockenperioden bestimmen, dann müssen wir die absolute monatliche Regenmenge berücksichtigen. Wir werden kaum wesentlich von den Tatsachen abweichen, wenn wir für die warmen Tropen für aride Monate einen Niederschlag unter $6 \mathrm{~cm}$ annehmen. Für die kalten Tropen liegt die Grenze bei $4 \mathrm{~cm}$. Legen wir unseren Berechnungen $6 \mathrm{~cm}$ zugrunde, dann werden wir eher zuviel als zu wenig aride Monate erhalten. Bezeichnen wir diese mit a und die Monate der Regenperiode mit ihren Anfangsbuchstaben, dann finden wir in Costa Rica, Mittelkolumbien und Flores nachstehende Trocken- und Regenperioden (Tab. 3).

Tab.3. - Aride und humide Monatc in Costa Rica, Mittelkolumbicn und Flores

\begin{tabular}{|c|c|c|c|c|c|c|c|c|c|c|c|}
\hline Ort & & & tate & & & & & & & & \\
\hline Caracolé & . & $\mathrm{J}$ & $\mathrm{F}$ & M & A & M & $\mathrm{J}$ & $\mathrm{J}$ & A & S & $\mathrm{O}$ \\
\hline San José & . & a & a & $a$ & $\mathrm{a}$ & $\mathrm{M}$ & J & $\mathrm{J}$ & A & S & C \\
\hline Cartago & . & a & $\mathrm{a}$ & a & $\mathrm{a}$ & M & $\mathrm{J}$ & J & A & $S$ & $\mathrm{O}$ \\
\hline Dagua & . & $\mathrm{J}$ & $\mathrm{F}$ & M & A & M & $\mathrm{J}$ & $\mathrm{J}$ & A & S & 0 \\
\hline Manizales & . & J & F & $\mathrm{M}$ & A & M & $\mathrm{J}$ & J & A & $S$ & $\mathrm{C}$ \\
\hline Cali & . & $\mathrm{J}$ & $\mathrm{F}$ & M & A & M & $\mathrm{J}$ & a & $\mathrm{a}$ & S & 0 \\
\hline Toetend & . & $\mathrm{J}$ & $\mathrm{F}$ & M & A & M & $\mathrm{J}$ & $\mathrm{J}$ & A & $S$ & ( \\
\hline Badjawa & - & $\mathrm{J}$ & $\mathrm{F}$ & M & A & M & a & $\mathrm{J}$ & $\mathrm{a}$ & $\mathrm{a}$ & a \\
\hline Reo & . & $\mathrm{I}$ & $\mathrm{F}$ & $\mathrm{M}$ & A & $\mathrm{a}$ & $\mathrm{a}$ & a & $\mathrm{a}$ & a & a \\
\hline
\end{tabular}

Wenn wir nach der absoluten monatlichen Regenmenge aride und humide Monate bestimmen, so lassen sich trockene (mit 12 ariden Monaten), wechselfeuchte (aride + humide Monate) und immerfeuchte (mit 12 humiden Monaten) Bereiche unterscheiden. Wir können aber LAUER nicht folgen, wenn er auf Grund dieser Klimabeurteilung von ariden und humiden Jahreszeiten spricht, weil dann trockene und immerfeuchte Klimate (Caracol, Dagua, Manizales und Tœtend) nur eine, sich über das ganze Jahr erstreckende Jahreszeit hätten! Er bestimmt Trocken- und Naßperioden, sagt aber nicht, ob die Jahreszeiten arid oder humid seien!

Die Berechnung der Regenverteilung in den Tropen nach der relativen Monatsregenmenge ergibt Regen- und Trockenzeiten, also die durch die geographische Breite bestimmten örtlichen Jahreszeiten. Die absolute Monatsregenmenge läßt aride und humide Monate bzw. Trocken- und Regenperioden erkennen, die durch die örtlichen Verhältnisse bestimmt sind.

Regenmengen. Es ist bekannt, daß bei gleicher Niederschlagsmenge eine höhere 'Temperatur ein trockeneres, eine niedrigere ein feuchteres Klima bedingt. Dieses ist offenbar eine Funktion von Regenmenge und Jahrestemperatur. R. LANG schlägt als solche den Regenfaktor $(\mathrm{RF})=$ Niederschlag $(\mathrm{N})$ : Jahrestemperatur $(\mathrm{T})$ vor. Damit lassen sich folgende Befeuchtungen unterscheiden: 1. arid (RF unter 40); 2 . semiarid ( $\mathrm{RF}$ 40-60); 3. semihumid (RF 60-100); 4. humid (RF 100-160) und 5. perhumid (RF über 160). Vom Regenfaktor N:T ist abgeleitet der Ariditätsindex $\mathrm{N}:(\mathrm{T}+10)$. Beide reduzieren die Niederschlagsmenge und gestatten so Klimavergleiche von Orten mit verschiedenen Regenmengen und Jahrestemperaturen.

Die Höhenklimate lassen sich durch die Befeuchtung (Regenfaktor, Ariditätsindex usw.) in Klimatypen unterteilen (vergl. S. 58!).

Parallelisieren. Wir haben gesehen, daß die durch Wende und Polarkreise geschiedenen Zonenklimate sich durch den Temperaturverlauf und Unterschiede in der Tageslänge unterscheiden. Sie kennzeichnen das Zonenklima. In den Tropen und in der gemäßigten Zone sinkt die mittlere Jahrestemperatur pro 100 Höhenzunahme um 
rund $0,6^{\circ} \mathrm{C}$. Wir dürfen daher tropische, gemäßigte und polare Höhenklimate nach der Jahrestemperatur unterscheiden. Maßgebend für die Befeuchtung ist die Jahresregenmenge und die Jahrestemperatur (Befeuchtung, Regenfaktor, Ariditätsindex usw.).

Die in der gemäßigten Zone bewährten Methoden dürfen wir auch auf die Tropen anwenden.

\section{DAS SYSTEM DER TROPENKLIMATE}

Aus obigen Tatsachen ergeben sich folgende zur Beurteilung des Tropenklimas maßgebende Gesichtspunkte:

I. Die Klimazone ist durch die geographische Breite bestimmt. Innerhalb der Wendekreise ist das Tropenklima gekennzeichnet: a) durch geringe jahreszeitliche und starke tägliche Temperaturschwankungen; b) annähernd ständige Tag- und Nachtgleiche; c) Regen- und Trockenzeiten als Jahreszeiten.

II. Die Klimazonen lassen sich durch die mittleren Jahrestemperaturen in Höhenklimate unterteilen. Für die Tropen ergeben sich folgende Höhengürtel (vergl. S. 57) : 1. Tierra caliente, 2. Tierra templada, 3. Tierra fría, 4. Páramo und 5. Tierra helada.

III. Die Höhenklimate lassen sich nach der Befeuchtung (vergl. S. 58) in 1. Aride, 2. Semiaride, 3. Semihumide, 4. Humide und 5. Perhumide Klimatypen unterteilen.

Kennen wir die geographische Breite, die Jahrestemperatur und die Regenmenge eines Ortes, so ist es möglich, den Klimatyp zu bestimmen, wie wir an drei Beispielen aus Kolumbien zeigen wollen. Tab. 4 und 5.

Tab. 4. - Klimadatcn von Bogotá, Medcllin und Bucnavcntura
\begin{tabular}{llccc} 
Ort & nördl. Breite & Regenmenge $(\mathrm{mm})$ & Temp. $\left({ }^{\circ} \mathrm{C}\right)$ & $\mathrm{RF}$ \\
\hline Bogotá & $4^{\circ}$ & 938 & 14 & 67 \\
Medellín & $6^{\circ}$ & 1550 & 21 & 73 \\
Buenaventura & $4^{\circ}$ & 7128 & 27 & 282
\end{tabular}

Tab. 5. - Klimaklassifikation von Bogotá, Medellin und Buenaventura

\begin{tabular}{llll} 
Kategorie & Bogotá & Medellin & Buenaventura \\
\hline I. Klimazone & tropisch & tropisch & tropisch \\
II. Höhenklima & $\begin{array}{l}\text { tierra fría } \\
\text { semihumid }\end{array}$ & semihumid & $\begin{array}{l}\text { terra calient } \\
\text { perhumid }\end{array}$ \\
III. Klimatyp & Kartoffeln & Kaffee, Bananen & Kautschuk \\
Kulturen & Getreide usw. & Zuckerrohr usw. & Palmen usw.
\end{tabular}

LAUER hat dieselben Klimate lediglich nach der Regenverteilung, d. h. nach der Anzahl von ariden und humiden Monaten beurteilt und kommt zum folgenden Ergebnis. Tab. 6.

Tab. 6. - Klima von Bogotá, Medellin und Bucnaventura von LAUER

\begin{tabular}{|c|c|c|c|c|c|}
\hline Ort & Meereshöhe (m) & Niedersch. $(\mathrm{mm})$ & $\begin{array}{c}\mathrm{M} \\
\text { aride }\end{array}$ & $\begin{array}{l}\text { ate } \\
\text { humide }\end{array}$ & Тур \\
\hline Bogotá & 2651 & 938 & 0 & 12 & Taet \\
\hline Medellín & 1538 & 1550 & o & 12 & Taefd \\
\hline Buenaventura & 12 & 7128 & o & 12 & Taefd \\
\hline
\end{tabular}

$\mathrm{Zu}$ dieser Klimaeinteilung von LAUER schreibt das Schweizerische Tropeninstitut in Basel: «Es wird hingewiesen auf die zunehmend genaueren Präzisierungen des Begriffes «Tropen», durch Wissmann, Maull, Troll und seine Schule, so insbesondere auf eine Arbeit von LAUER über die Gliederung der vertikalen Klimazonen an den Beispielen Südamerikas und Afrikas. Unserem Experten erscheint die Abgrenzung in aride und humide Zonen und die Einführung der Isohygromenen eine glückliche Konzeption, die sich pflanzengeographisch und auch hinsichtlich der landwirtschaftlichen Nutzung eines Gebietes als außerordentlich wertvoll erweist». 
Man wird dem Basler Tropeninstitut nur beistimmen können, wenn es von einer Klimasystematik der Tropen verlangt, daß sie die Höhenklimate erkennen lasse und Schlüsse auf die landwirtschaftliche Nutzung gestatte. Nun kennen wir die Klimaklassifikationen von Bogotá, Medellín und Buenaventura nach den Regenfaktoren und von LAUER. Weiter kennen wir die landwirtschaftliche Nutzung dieser Regionen und ihre Meereshöhe. Wir können daher leicht nachprüfen, welcher dieser beiden Vorschläge die vom Schweizerischen Tropeninstitut gestellten Forderungen erfüllt (Tab. 7).

Tab.7. - Vergleich der Klimatypen nach den Regenfaktoren und ron LaUER

Ort und Höhe $(m)$ Regenfaktoren LaUer Kulturen

Bogotá (2651) tropisch, tierra fría, semihumid Taefd Kartoffeln, Getreide

Medellín (1538) tropisch, tierra templada, semihumid Taefd Kaffee, Bananen, Zuckerrohr Buenaventura (12) tropisch, tierra caliente, perhumid Taefd Kautschuk, Palmen

Daß der LAUER'sche Klimatyp Taefd keine Schlüsse auf die Höhenlage und die landwirtschaftliche Nutzung gestattet, bedarf kaum weitere Beweisführung! Die Regenfaktoren N:T lassen durch die $\mathrm{T}$ die Höhenlage und durch den Quotienten die Befeuchtung erkennen und Bogotá, Medellín und Buenaventura gehören verschiedenen Klimatypen an. Wir erhalten so folgendes

$$
\text { Klimasystem der Tropen }
$$

Höhenklimate Klimatypen (nach Regenfaktoren)

\begin{tabular}{|c|c|c|c|c|c|}
\hline & unter 40 & $40-60$ & $60-100$ & $100-160$ & über 160 \\
\hline $\begin{array}{l}\text { Tierre helada } \\
\text { Páramo } \\
\text { Tierra fria }\end{array}$ & $\begin{array}{l}\text { arid } \\
\text { arid } \\
\text { arid }\end{array}$ & $\begin{array}{l}\text { semiarid } \\
\text { semiarid } \\
\text { semiarid }\end{array}$ & $\begin{array}{l}\text { semihumid } \\
\text { semihumid } \\
\text { semihumid } \\
\text { (Bogotá) }\end{array}$ & $\begin{array}{l}\text { humid } \\
\text { humid } \\
\text { humid }\end{array}$ & $\begin{array}{l}\text { perhumid } \\
\text { perhumid } \\
\text { perhumid }\end{array}$ \\
\hline Tierra templada & arid & semiarid & $\begin{array}{l}\text { semihumid } \\
\text { (Medellin) }\end{array}$ & humid & perhumid \\
\hline Tierra caliente & arid & semiarid & semihumid & humid & $\begin{array}{l}\text { perhumid } \\
\text { (Buenaventura) }\end{array}$ \\
\hline
\end{tabular}

Die 'Tropen kennzeichnen somit 25 Klimatypen, aus denen wir die Höhendifferenzierung leicht erkennen können. Jedem Typ entspricht auch eine bestimmte landwirtschaftliche Nutzung und die obige Klimaunterteilung wird denn auch von den südamerikanischen Landwirten in der Praxis berücksichtigt.

\section{ZUSAMMENFASSUNG}

Bei der Systematik des Tropenklimas sind zu berücksichtigen: 1. Die geographische Breite, die das Zonenklima bestimmt. Hieraus lassen sich jährlicher Temperaturablauf, die Schwankungen der Tageslänge und die Jahreszeiten erfassen. 2. Die Höhenlage bestimmt über die mittlere Jahrestemperatur innerhalb der Zone das Höhenklima. In den Tropen: tierra helada, páramo, tierra fría, tierra templada und terra cäliente. 3. Die Befeuchtung bestimmt innerhalb des Höhenklimas den Klimatyp. Die Regenfaktoren 40, 60, 100 und 160 ergeben aride, semiaride, semihumide, humide und perhumide Klimatypen.

In den Tropèn Südamerikas haben sich die Regenfaktoren bewährt, weil 1. die Klimazone als gegeben vorausgesetzt ist; 2. der Nenner (Temperatur) zur Bestimmung des Höhengürtels verwendet wird; 3. der Quotient (N:T) die Befeuchtung innerhalb des Höhengürtels angibt. Die Regenfaktoren versagen, wenn nur der Quotient ( $\mathrm{N}: \mathrm{T}$ ) gebraucht wird, weil dann das Höhenklima nicht bestimmt wird. Beim Vergleich von Gebieten gleicher Regenfaktoren muß die Höhe und die Zone berücksichtigt werden.

So haben die Regenfaktoren bald sehr gute, bald unbefriedigende Resultate gegeben. Sobald wir aber einmal wissen, wie der LANG'sche Regenfaktor anzuwenden ist, dürfte die Diskussion bald beendet sein. 


\section{LITERATURHINWEISE}

KNoch, K. \& Schulze, A.: 1952. Methoden der Klimaklassifikation. Petermanns Geogr. Mitt. Ergänzungsheft Nr. 249. - LANG, R.: 1915. Versuch einer exakten Klassifikation der Böden in klimatischer und geologischer Hinsicht. Int. Mitt. f. Bodenkunde. - LAUeR, W.: 1952. Humide und aride Jahreszeiten in Afrika und Südamerika und ihre Beziehung zu den Vegetationsgürteln. Bonner Geogr. Abhandlg. Heft 9. - Schaufelberger, P.: 1952. Zur Gliederung des tropischen Klimas. Geographica Helv. VII/2. Ders. 1955. Vierzig Jahre Regenfaktor. Vierteljahrsschr. d. Naturf. Gesellschaft in Zürich. 10o. - Schweizerisches Tropeninstitut 1956: Briefl. Mitteilung.

\section{A PROPOS DE LA SYSTÉMATIQUE DES CLIMATS TROPICAUX}

Dans une classification des climats tropicaux, il faut considérer: 1) la latitude qui détermine les variations de la température, les différentes longueurs des jours, les saisons et, de là, le climat de la zone; 2) la température moyenne annuelle d'après laquelle on distingue les «climats d'altitude» (tierra helada, páramo, tierra fría, templada, caliente); 3 ) l'humidité qui conduit à une nouvelle subdivision parmi les climats d'altitude en type aride, semiaride, semi-humide, humide et perhumide. Le système compte ainsi 25 types ou régions climatiques. Dans les tropiques de l'Amérique du sud, ce sont les facteurs de pluviosité qui ont fait leurs preuves, car 1) la zone climatique est donnée, 2) on utilise le dénominateur (température $\mathrm{T}$ ) pour déterminer les zones d'altitudes, 3) le quotient $(\mathrm{N}: \mathrm{T})$ donne l'humidité à l'intérieur des zones d'altitude. Par contre, les facteurs de pluviosité ne sont d'aucune utilité lorsque le quotient seul est utilisé, parce que dans ce cas, le climat d'altitude n'est pas fixé.

\section{DER XVIII.INTERNATIONALE GEOGRAPHENKONGRESS UND DIE IX. GENERALVERSAMMLUNG DER INTERNAT. GEOGRAPHISCHEN UNION (IGU) IN RIO DE JANEIRO I $956^{*}$ OtMar WidMer}

Der Geographenkongreß, der erstmals südlich des Äquators und in den Tropen stattfand, wurde vom 9. bis 18. August 1956 in Brasilien abgehalten, in der prächtigen, über 3 Millionen Einwohner zählenden Bundeshauptstadt an der GuanabaraBucht am Fuße des Pão de Açúcar ( $Z$ Zuckerhut»), in Rio de Janeiro, der schönst gelegenen Stadt der Erde.

Sie war am letzten Kongre $\beta$ in Washington 1952, in Konkurrenz stehend mit Edinburgh und Wien, als Tagungsort gewählt worden. Der mit der Durchführung betraute «Conselho Nacional de Geografia» (Nationalkomitee für die IGU) hatte ein Organisationskomitee eingesetzt, in dem zufolge seines neu übernommenen Amtes als Präsident des «Instituto Brasileiro de Geografia e Estatistica» Prof. Ing. Jurandyr Pires Ferreira das Präsidium führte und dem als Vizepräsident Prof. Fábio de Macedo Soares Guimaraes und als Generalsekretär, dem die große Vorbereitungsarbeit oblag, Prof. der Geographie an der Universität von Brasilien in Rio Hilgard O'Reilly Sternberg angehörten. Durch Zirkular vom Dezember 195t und Mai 1956 waren die Geographen der ganzen Welt zur Teilnahme eingeladen worden; das Programm war vom Exekutivkomitee der Internationalen Geographischen Union (IGU) an der Tagung im Makerere College, Kampala, Uganda, im September 1955 genehmigt worden. Die brasilianischen Marinebehörden hatten die Räume der Escola Naval für Sitzungen und Unterkunft zur Verfügung gestellt. Diese Marineschule liegt auf der durch den Franzosen de Villegaignon 1555 befestigten und nach ihm benannten kleinen Insel und ist durch eine Brücke mit dem aufgeschütteten Lokalflughafen «Santos Dumont» der Stadt Rio verbunden, die als Ausgangslager auf dem Festland zwecks Belagerung der Insel 1565 von Estácio de Sá gegründet und 1763 an Stelle von Bahia als Hauptstadt gewählt worden ist.

Der Kongreß begann Donnerstag, 9. August, vormittags mit einem Besuch beim Staatspräsidenten Dr. Juscelino Kubitschek de Oliveira, beim Außenminister Botschafter José Carlos de Macedo Soares und beim Präfekten des Bundesdistrikts von Rio Dr. Francisco Negrao de Lima. Nach großartigem Empfang im Palácio

* Referat, gehalten in der Sektion «Geographie und Kartographie» an der SNG-Tagung in Basel am 23. September 1956. 